\title{
Right Vertebral Artery Stenosis with Left Hemispheric TIA: A Perplexing Etiology
}

Charles Hartranft*, Seth Noland, Aaron Kulwicki and Thomas Hartranft

Department of Surgery, Mount Carmel Health System, Columbus, Ohio, USA

\begin{abstract}
A 59-year-old female presented with symptoms concerning for left hemispheric transient ischemic attacks. Multiple imaging modalities confirmed the presence of acute left sided cerebral infarcts along with complete left internal carotid artery occlusion. Following discharge on appropriate medical therapy, the patient represented weeks later with similar complaints. Angiography confirmed left internal carotid artery occlusion and identified a right vertebral artery supplying the entire contralateral hemisphere. A segment of severe stenosis at the vertebral artery origin was identified and stented. Post-operatively, the patient recovered well and was discharged home without significant neurological deficits. During follow-up, in-stent restenosis developed at 7 months requiring additional intervention with balloon angioplasty and re-stenting. To date the patient suffers no significant neurologic deficits, is maintained on maximal medical therapy, and continues follow up with serial exams and ultrasound imaging.
\end{abstract}

Keywords: Vertebral artery stenosis; Vertebral artery stenting; Vertebrobasilar insufficiency

\section{Introduction}

Vertebral Artery (VA) atherosclerosis is a seldom-discussed disease that contributes significantly to posterior circulation ischemic events. Demonstrated in up to $40 \%$ of individuals with cerebrovascular disease, VA atherosclerosis accounts for nearly $30 \%$ of all transient ischemic attacks (TIA) or stroke [1]. Historically, less than 5\% of surgeries performed for cerebrovascular disease address lesions of the VA origin where atherosclerotic disease is most prevalent [2]

First studied in the 1960's, the natural history of VA occlusive disease was felt to be benign when compared with carotid distribution ischemic events [3]. It is not until recently that current investigators are beginning to challenge this notion. In a literature review, Flossmann and Rothwell found no evidence that patients presenting with posterior circulation ischemic events were at lower risk of subsequent stroke or death when compared to those presenting with carotid TIA or minor stroke. Furthermore, their risk of stroke was slightly higher in the acute time period following initial symptom onset [4]. A complicating issue is the fact several other medical conditions may produce symptoms mimicking posterior circulation ischemic events such as use of antihypertensive medications, cardiac arrhythmias, anemia, and benign vertigo.

Unlike carotid intervention, where well-defined roles for secondary prevention of ischemic events exist, the benefit of surgical or endovascular revascularization for proximal VA atherosclerosis remains yet to be clearly defined. Appropriate patient selection for

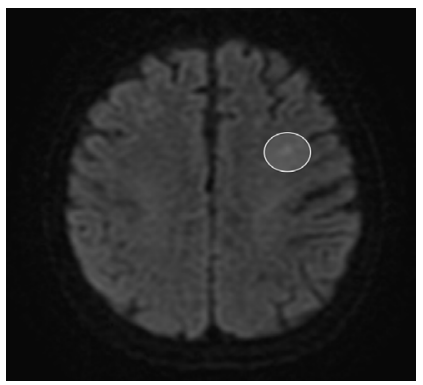

Figure 1: Magnetic resonance (MR) angiography of the head depicting acute left hemispheric subcortical infarction. intervention requires individualized assessment of risks balanced with potential benefits of revascularization [5]. This report presents a patient with recurrent left hemispheric TIA as a result of right VA stenosis managed via endovascular intervention.

\section{Case Report}

A 59 year-old female was evaluated in the in-patient setting for recent complaints of intermittent right upper and lower extremity weakness. Symptoms had increased in frequency and were also associated with new onset speech difficulties. Pertinent medical history included hypertension well controlled with use of carvedilol and losartan. She endorsed no history of dyslipidemia, coronary artery disease, or diabetes. Social history was remarkable for 49 packyear nicotine use and a family history of maternal Peripheral Arterial Disease (PAD) was noted. Upon evaluation by the vascular surgery service, symptoms had completely resolved and physical examination was unremarkable. Pertinent serum laboratory analysis was also unremarkable for anemia, hyperglycemia, renal insufficiency, and electrolyte abnormalities. Additionally, non-fasting lipid panel results were as follows: Total cholesterol $122 \mathrm{mg} / \mathrm{dL}$; Triglycerides $82 \mathrm{mg} /$ $\mathrm{dL}$; HDL $59 \mathrm{mg} / \mathrm{dL}$; LDL $47 \mathrm{mg} / \mathrm{dL}$. Prior to consultation, imaging studies obtained included Magnetic Resonance (MR) and Computed Tomography (CT) angiography of the head and neck. MR imaging revealed high-grade stenosis of the left Internal Carotid Artery (ICA) and small bilateral subcortical infarctions (Figure 1). Additionally, CT displayed complete left ICA occlusion, 50\% right ICA stenosis, and multi-segment disease of the left Vertebral Artery (VA) (Figure 2). Carotid duplex ultrasonography was performed which confirmed left ICA occlusion, moderate right ICA stenosis, and visualized bilateral VA antegrade blood flow (Figure 3). The patient was discharged home on clopidogrel, statin therapy, and previous anti-hypertensive medications.

*Corresponding author: Charles Hartranft, Department of Surgery, Moun Carmel Health System, 793 West State St, OH 43222, Columbus, USA, Tel: 330-518-9400; E-mail: charles.hartranft@gmail.com

Received June 12, 2014; Accepted July 21, 2014; Published July 23, 2014

Citation: Hartranft C, Noland S, Kulwicki A, Hartranft T (2014) Right Vertebral Artery Stenosis with Left Hemispheric TIA: A Perplexing Etiology. J Vasc Med Surg 2: 144. doi: 10.4172/2329-6925.1000144

Copyright: (c) 2014 Hartranft C, et al. This is an open-access article distributed under the terms of the Creative Commons Attribution License, which permits unrestricted use, distribution, and reproduction in any medium, provided the original author and source are credited. 


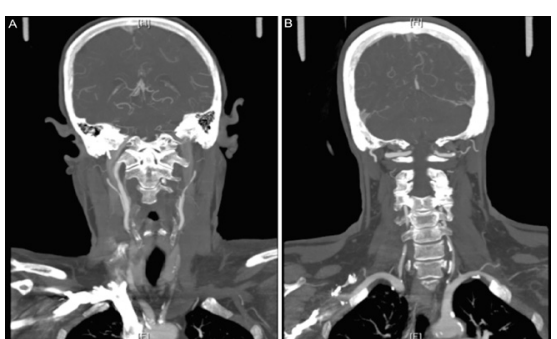

Figure 2: Computed tomography (CT) angiography of neck depicting (A) complete left ICA occlusion, 50\% right ICA stenosis, and (B) multi-segment disease of the left vertebral artery (VA).

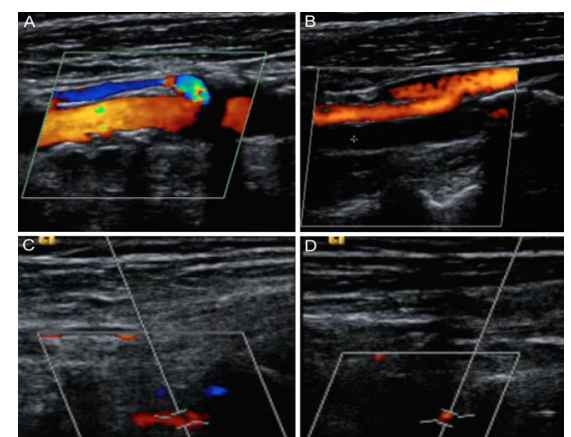

Figure 3: Carotid duplex ultrasonography depicting $(A)$ moderate right ICA stenosis, (B) left ICA occlusion, (C) right VA antegrade flow, and (D) left VA antegrade flow.

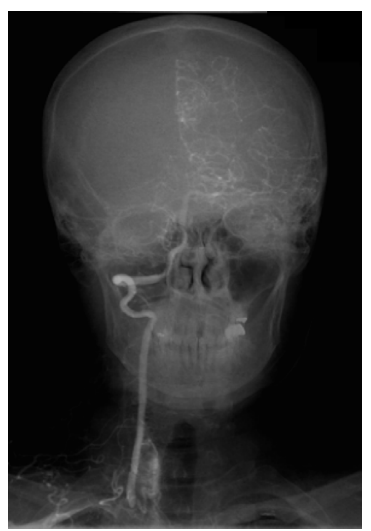

Figure 4: Angiogram displaying severe right VA stenosis with contralateral filling of the left cerebral hemisphere.

Follow up was established for right ICA stenosis and PAD risk factor modification was strongly encouraged.

She represented one month later with similar symptomatology suggestive of left hemispheric TIA. Angiography confirmed left ICA occlusion, moderate right ICA stenosis, and diffuse left VA disease. Interestingly, a high grade (90\%) stenosis was identified at the origin of the right VA supplying blood flow to the entire contralateral cerebral hemisphere (Figure 4). A $5 \mathrm{~mm} 30 \mathrm{~mm}$ PRECISE (Cordis, Bridgewater, NJ, USA) stent was placed utilizing an embolic protection device (Figure 5). The patient suffered no immediate complications and was discharged home with appropriate follow-up.
Post-operative right VA duplex surveillance at three, six, and seven months demonstrated increasing Peak Systolic Velocities (PSVs) in excess of $300 \mathrm{~cm} / \mathrm{sec}$ consistent with in-stent restenosis. Reintervention was performed by placement of a $5 \mathrm{mmx} 39 \mathrm{~mm}$ iCast (Atrium, Hudson, NH, USA) stent with use of an embolic protection device. To date, outpatient follow-up with arterial duplex surveillance at three months displays PSVs of $<100 \mathrm{~cm} / \mathrm{sec}$ and the patient remains symptom free. She has been provided with smoking cessation materials and education however unfortunately continues to smoke.

\section{Discussion}

Low-flow hemodynamics and embolic disease are the two mechanisms responsible for vertebrobasilar circulation ischemic events. Although fewer patients suffer from embolic disease than from hemodynamic mechanisms, actual infarctions in the posterior circulation are most commonly the result of embolic events [6]. There is a lack of randomized controlled data regarding the medical management of asymptomatic VA atherosclerosis. Current guidelines from several vascular, neurosurgical, and multidisciplinary medical societies recommend best medical management to include those practices established for carotid artery disease [7]. Intervention for disease, whether surgical or endovascular, takes into account symptomology, concurrent carotid artery disease, circle of Willis patency, and contralateral posterior circulation anatomy in order to guide treatment strategies. In addition to carotid artery atherosclerosis affecting anterior circulation, Ciccone and colleagues have extensively studied carotid artery dolichoectasias in terms of clinical significance. This rare entity, found in $2-6 \%$ of the general population, was studied for association with neurological symptomatology in patients without concomitant carotid artery atherosclerotic disease. Investigators found carotid echo-color Doppler to be both sensitive and specific for diagnosis. Additionally, five year follow up revealed carotid artery tortuosity and kinking to be a progressive pathology with tortuosity specifically, associated with an increased risk of cardiovascular death in the study population [8]. Similar research demonstrated a positive correlation between carotid artery dolichoectasia and arterial hypertension in a study population with non-ischemic dilated cardiomyopathy [9].

Established indications for intervention of extracranial VA atherosclerosis include symptomatic high-grade stenosis, as well as

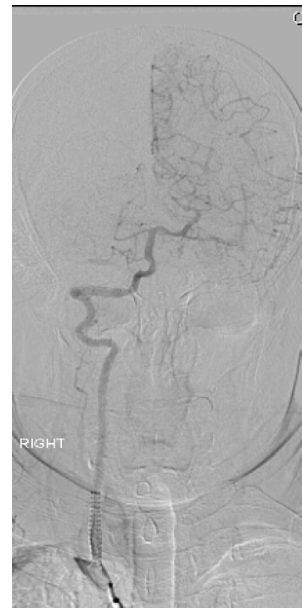

Figure 5: Angiogram displaying technical success of $5 \mathrm{~mm} \times 30 \mathrm{~mm}$ stent placement across proximal VA lesion C. 
Citation: Hartranft C, Noland S, Kulwicki A, Hartranft T (2014) Right Vertebral Artery Stenosis with Left Hemispheric TIA: A Perplexing Etiology. J Vasc Med Surg 2: 144. doi: 10.4172/2329-6925.1000144

Page 3 of 3

progressive, high-grade stenosis in the presence of compromised contralateral VA flow [10]. No randomized controlled studies comparing open versus endovascular treatment of VA atherosclerosis currently exist. The largest retrospective study available comparing the two modalities found open intervention (VA to common carotid artery transposition) to provide better symptomatic relief in those presenting with transient symptoms attributed to low-flow hemodynamics. Although not statistically significant, trends toward superior longterm patency along with decreased need for secondary intervention were also observed in the open group. Open intervention, as expected, was associated with higher rates of perioperative morbidity [11]. More recently however, treatment strategies have shifted toward endovascular approaches to include VA stenting.

A large review of over 700 patients with proximal VA atherosclerosis undergoing VA stenting demonstrated the efficacy and safety of this growing treatment modality. Technical success and perioperative event rates of $99 \%$ and $1.5 \%$ respectively, were observed in symptomatic patients treated. With follow up of nearly two years, many case series included in this review demonstrated In-Stent Restenosis (ISR) as the primary limitation of endovascular intervention when compared with the durable outcomes of open surgical intervention [12]. Atherosclerotic lesion length has been well recognized and validated as an important predictor of ISR in cardiac literature. Currently, smaller studies evaluating lesion length in the VA have also found it an independent predictor of ISR [13]. Higher elastic recoil intrinsic to VA anatomy has been another hypothesized explanation for such high rates of ISR observed with endovascular intervention. As many case series and reviews in the literature allude to, VA atherosclerosis can be managed safely and efficaciously with endovascular techniques. Investigators have now turned attention to technical aspects to improve the durability of endovascular intervention.

Balloon expandable coronary stents appear to be the most common stent employed in several case series for treatment of proximal VA atherosclerosis. A large prospective study from 2003 to 2011 evaluated Drug Eluting Coronary Stents (DES) versus Bare Metal Coronary Stents (BMS) for superiority when used to treat proximal VA atherosclerosis. Over 200 patients were treated for symptomatic lesions of greater than $70 \%$ stenosis. With long term follow up of more than 3.5 years, both DES and BMS use was found to be safe and technically feasible. While both effective for symptomatic treatment of VA atherosclerosis, DES use significantly decreased the need for later endovascular intervention in the long term [14].

In summary, VA atherosclerosis is an underappreciated disease process that contributes significantly to cerebrovascular disease. In general, treatment with open intervention carries a higher morbidity and mortality in the acute setting while appearing to be more durable in terms of long-term patency. While the literature supports the safety and feasibility of VA stenting, it is plagued by ISR in those with long atherosclerotic lesions. When stenting is performed, use of coronary drug eluting stents appears to be favored. Decisions to intervene, whether open or endovascular takes into account contralateral VA flow, symptomatology, and concomitant anterior circulation occlusive disease.
This case demonstrates a scenario in which a patient suffered TIA secondary to proximal VA atherosclerosis. Aberrant anatomy complicated the case leading to a delayed diagnosis prior to endovascular treatment. Her post-operative course was complicated by ISR mandating further endovascular therapy. This review highlights the need for increased awareness of posterior circulation ischemic events as well as treatment modalities employed for intervention.

\section{References}

1. Vemmos K, Takis C, Georgilis K, Zakopoulos NA, Lekakis JP, et al. (2000) The Athens Stroke Registry: Results of a Five-Year Hospital-Based Study. Cerebrovasc Dis 10: 133-141.

2. Imparato AM (1985) Vertebral Arterial Reconstruction: A Nineteen-Year Experience. J Vasc Surg 2: 626-634.

3. Marshall $\mathrm{J}$ (1964) The natural history of transient ischemic cerebro-vascular attacks. Q J Med 33: 309-324.

4. Flossmann E and Rothwell PM (2003) Prognosis of vertebra-basilar transient ischemic attack and minor stroke. Brain 126: 1940-1954.

5. Brasiliense LB, Albuquerque F, Spetzler R, Hanel R (2014) Advances and Innovations in Revascularization of Extracranial Vertebral Artery. Neurosurgery 74: 102-115.

6. Caplan LR, Wityk RJ, Glass TA, Tapia J, Pazdera L, et al. (2004) New England Medical Center Posterior Circulation Registry. Ann Neurol 56: 389-398.

7. Brott T, Halperin J, Abbara S, Bacharach M, Barr J, et al. (2013) Guidelines on the Management of Patients with Extracranial Carotid and Vertebra Artery Disease: Executive Summary. Catheterization and Cardiovascular Interventions 81: E75-E123.

8. Ciccone MM, Sharma RK, Scicchitano P, Cortese F, Salerno C, et al. (2014) Dolichocarotids: Echo-Color Doppler Evaluation and Clinical Role. J Atheroscler Thromb 21: 56-63.

9. Ciccone MM, Scicchitano P, Palumbo V, Cortese F, Valecce R, et al. (2012) Dolichocarotids and dilated cardiomyopathy: Is there a relationship? Int J Cardiol 158: 123-125.

10. Ernemann U, Bender B, Melms A, Brechtel K, Kobba J, et al. (2012) Current Concepts of the Interventional Treatment of Proximal Supraaortic Vesse Stenosis. Vasa 41: 313-318.

11. Shutze W, Gierman J, McQuade K, Pearl G, Smith B (2014) Treatment of proximal vertebral artery disease. Vascular 22: 85-92

12. Stayman AN, Nogueira RG, Gupta R (2011) A Systematic Review of Stenting and Angioplasty of Symptomatic Extracranial Vertebral Artery Stenosis. Stroke 42: $2212-2216$

13. Lin YH, Liu YC, Tseng WY, Juang JM, Hung CS, et al. (2006) The Impact of Lesion Length on Angiographic Restenosis After Vertebral Artery Origin Stenting. Eur J Vasc Endovasc Surg 32: 379-385.

14. Song L, Li J, Gu Y, Yu H, Chen B, et al. (2012) Drug-Eluting vs. Bare Metal Stents for Symptomatic Vertebral Artery Stenosis. J Endovasc Ther 19: 231238 . 